\title{
ZERO SQUARE NEAR-RINGS
}

\author{
PATRICIA JONES
}

(Received 27 February 1990)

Communicated by B. J. Gardner

\begin{abstract}
The purpose of this paper is to provide examples and explore properties of a wide variety of zero square (left) near rings. Among the main results are complete classifications of (i) finite Abelian groups which are the additive group of a zero square near-ring and (ii) finite non-Abelian groups which support 3-nilpotent distributive zero square near-rings.
\end{abstract}

1991 Mathematics subject classification (Amer. Math. Soc.) 16 A 76.

\section{Introduction and preliminaries}

Zero square near-rings having both distributive properties were considered by Heatherly in [3]. He gave examples, explored nilpotency and properties of the additive groups of such near-rings, and raised the question of whether all zero square near-rings are right distributive. The present author [5] answered Heatherly's question by giving an example of a non-distributive zero square near-ring on the dihedral group of order eight. More recently, Feigelstock [1] has provided several examples of both Abelian and non-Abelian zero square near-rings which are not right distributive.

In this paper we will show that there is an abundance of zero square nearrings (distributive, pseudo-distributive, and neither) having a wide variety of additive groups. Complete classifications are given for finite Abelian groups which are the additive group of a zero square near-ring, and for non-Abelian groups which support 3-nilpotent distributive zero square near-rings. We also show that any zero square near-rings with cyclic addition is 3-nilpotent, and

(C) 1991 Australian Mathematical Society 0263-6115/91 \$A2.00+0.00 
we provide several necessity conditions for a non-distributive distributively generated zero square near-ring.

Throughout the paper $Z S$ near-ring will denote a left near-ring, which is not a ring, in which $x^{2}=0$ for all $x$ and $x y \neq 0$ for some $x y$. These basic properties of such a near-ring are trivial to verify.

LEMMA 1.1. If $N$ is a $Z S$ near-ring, then

(i) $N$ is zero-symmetric;

(ii) $x y x=0$ for all $x, y \in N$;

(iii) If $x \neq 0$ and $y \neq 0$, then $x y \neq x$ and $x y \neq y$.

If $N$ is a near-ring and $x \in N, x$ is called a right-distributive element if $(a+b) x=a x+b x$ for every $a, b \in N . N$ is a distributive near-ring if all its elements are right distributive, and $N$ is distributively generated (d.g.) if $N^{+}$is generated by a set of right distributive elements [7]. $N$ is pseudo-distributive if $(a b+c d) x=a b x+c d x$ and $a b+c d=a b$ for all $a, b, c, d, x \in N$ [4]. A near-ring is Abelian if its additive group is Abelian.

\section{Abelian zero square near-rings}

It is well known that distributive or distributively generated near-rings with Abelian additive groups are rings. But pseudo-distributive near-rings which are not rings can be Abelian. In this section we classify finite Abelian groups which support non-pseudo-distributive $Z S$ near-rings. We also show that every $Z S$ near-rings with cyclic addition is 3-nilpotent, and give examples of both pseudo-distributive and non-pseudo-distributive $Z S$ near-rings defined on cyclic and non-cyclic groups.

THEOREM 2.1. The cyclic group of order $n$ is the additive group of a ZS near-ring if and only if $n=p^{2} m$ for some prime $p$ and $m>1$.

Proof. (i) Let $N$ be a $Z S$ near-ring of order $n$ with $N^{+}=\langle x\rangle$. Since $N^{2} \neq 0$ and $x(m x)=0$ for every $m \in Z$, there exist distinct positive integers $j, k<n$ such that $(j x) x=k x$. Then $(j x)(j x)=j k x=0$. So $n \mid j k$. Also notice that

$$
[(j x)(j x)] x=0=(j x)[(j x) x]=(j x)(k x)=k^{2} x .
$$

This implies $n \mid k^{2}$. But $n+k$; hence $n$ is not square-free.

Now suppose $n=p^{2}$. Since $p|j, p| k, j<p^{2}$, and $k<p^{2}$, we can write $j=a p$ and $k=b p$ for positive integers $a, b<p$. Therefore, there 
exists a positive integer $c<p$ such that $b c \equiv 1(\bmod p)$. Let $c b=r p+1$ for $0 \leq r<p$. Then

$$
(a p x)(c a x)=c a b p x=(r p+1)(a p x)=r p^{2} a x+a p x=a p x,
$$

which gives us the contradiction

$$
[(a p x)(\operatorname{cax})](\operatorname{cax}) \neq(a p x)[(\operatorname{cax})(\operatorname{cax})] .
$$

(ii) Let $n=p^{2} m$ for some prime $p$ and some integer $m>1$, and let $N^{+}=\langle x\rangle$ be the cyclic group of order $n$. Define multiplication on $N^{+}$by

$$
(p x)(j x)=j p m x \text { and }(r x)(j x)=0 \text { for } r \neq p .
$$

It is routine to check that $(N,+, \cdot)$ is a $Z S$ near-ring.

The near-rings constructed in part (ii) above are pseudo-distributive and 3-nilpotent. We do not know whether all $Z S$ near-rings with cyclic addition are pseudo-distributive, but they are all 3-nilpotent.

THEOREM 2.2. Every ZS near-ring with cyclic addition is 3-nilpotent.

Proof. Let $N$ be a $Z S$ near-ring of order $n$ with $N^{+}=\langle x\rangle$. Suppose $(j x)(k x)(v x) \neq 0$ for positive integers $j, k, v<n$. Then $(j x) x=t x \neq 0$ and $(k x) x=m x \neq 0$. Hence $(j x)(t x)=t^{2} x=0$ and $(k x) x=m x \neq 0$. Hence $(j x)(t x)=t^{2} x=0$ and $(k x)(m x)=m^{2} x=0$, which implies $n \mid t^{2}$ and $n \mid m^{2}$. Therefore, $n \mid t m$, giving us the contradiction

$$
(j x)[(k x)(v x)]=(j x)(v m x)=t v m x=0 .
$$

We now consider Abelian $Z S$ near-rings with non-cyclic addition.

LEMMA 2.3. Let $p$ and $q$ be distinct primes. If $N \cong Z_{p^{a}} \oplus Z_{p^{2}}$ for $1 \leq$ $a \leq 2, N \cong Z_{p} \oplus Z_{p q}$, or $N \cong Z_{p} \oplus Z_{p} \oplus Z_{p}$, then $N$ is the additive group of a $Z S$ near-ring.

Proof. (i) If $N=\langle x\rangle \oplus\langle y\rangle$ where $\langle x\rangle$ has order $p^{\alpha}$ and $\langle y\rangle$ has order $p^{2}$, define multiplication on $N$ by

$$
(x+y)(j x+k y)=k p y \quad \text { and } \quad a b=0 \text { if } a \neq(x+y) .
$$

(ii) If $N=\langle x\rangle \oplus\langle y\rangle$ where $\langle x\rangle$ has order $p$ and $\langle y\rangle$ has order $p q$, let $t$ be the smallest positive integer such that $p \mid(q+t)$. Define multiplication on $N$ by

$$
(x+y)(j x+k y)=t(j-k) x-q(j-k) y \quad \text { and } \quad a b=0 \text { if } a \neq(x+y) .
$$


(iii) If $N=\langle x\rangle \oplus\langle y\rangle \oplus\langle z\rangle$ where each summand has order $p$, define multiplication on $N$ by

$$
x(j x+k y+m z)=k z \text { and } a b=0 \text { if } a \neq x .
$$

It is routine to verify that for each of these multiplications $(N,+, \cdot)$ is a $Z S$ near-ring.

THeOREM 2.4. A non-cyclic Abelian group $N$ is the additive group of a ZS near-ring if and only if $N$ is not isomorphic to $Z_{p} \oplus Z_{p}$ for some prime $p$.

Proof. (i) If $N$ is not isomorphic to $Z_{p} \oplus Z_{p}$, then $N$ has a direct summand $G$ such that $G \cong Z_{p^{2} m}$ for $m>1, G \cong Z_{p^{\alpha}} \oplus Z_{p^{2}}$ for $1 \leq \alpha \leq 2$, $G \cong Z_{p} \oplus Z_{p q}$, or $G \cong Z_{p} \oplus Z_{p} \oplus Z_{p}$. It follows from Theorem 2.1 and Lemma 2.3 that $G$ supports a $Z S$ near-ring. Therefore, the direct sum of the $Z S$ near-ring on $G$ and the zero ring on $N / G$ is a $Z S$ near-ring with additive group isomorphic to $N$.

(ii) Suppose $N$ is a zero square near-ring and $N^{+}=\langle a\rangle \oplus\langle b\rangle$, where each summand has order $p$.

If $(j a) a=r a+s b$ for some positive integer $j<p$ and non-negative integers $r, s<p$, then $(j a)(j a)=j r a+j s b=0$. Therefore, $r=s=0$. Now suppose $(j a) b=r a+s b$. Then

$$
(j a)(r a+s b)=s r a+s^{2} b=0 .
$$

Hence $r=s=0$. By a similar argument, it can be shown that $(j b) b=$ $(j b) a=0$. But since $N$ does not have zero multiplication, there exist positive integers $j, k<p$ and non-negative integers $v, w<p$ such that $(j a+k b)(v a+w b) \neq 0$. It follows that $(j a+k b) a=m a+n b \neq 0$ or $(j a+k b) b=r a+s b \neq 0$ for non-negative integers $m, n, r, s<p$.

If $m a+n b=0$, then

$$
(j a+k b)^{2}=(j a+k b)(k b)=k r a+k s b=0
$$

which implies $r=s=0$. This contradiction gives us $m a+n b \neq 0$. The supposition that $r a+s b=0$ results in the same contradiction. Therefore, $m a+n b \neq 0$ and $r a+s b \neq 0$.

If $p=2$, then $j=k=m=n=r=s=1$. But this implies that $(a+b) a=a+b$, which is impossible. So $p>2$.

Notice that

$$
(j a+k b)(m a+n b)=\left(m^{2} r+n r\right) a+(m n+n s) b=0,
$$

and

$$
(j a+k b)^{2}=(j m+k r) a+(j n+k s) b=0 .
$$


Hence $m+s \equiv 0(\bmod p), m^{2}+n r \equiv 0(\bmod p)$, and $j m+k r \equiv 0(\bmod p)$.

Since $p>2$, there exists a positive integer $c<p$ such that $c \not \equiv$ $k n^{-1}(\bmod p)$. So

$$
(j m+k r) \equiv c\left(n r+m^{2}\right) \equiv 0(\bmod p),
$$

and

$$
j m-c m^{2} \equiv c n r-k r \equiv(j-c m) m \equiv(c m-j) s \equiv(c n-k) r(\bmod p) .
$$

Therefore, $(c m-j) r^{-1} \equiv(c n-k) s^{-1} \not \equiv 0(\bmod p)$.

$$
\text { Let }(j-c m) r^{-1} \equiv d \equiv(k-c n) s^{-1}(\bmod p) \text {. Now notice that }
$$

$$
\begin{aligned}
(j a+k b)(c a+d b) & =(c m+d r) a+(c n+d s) b \\
& =\left[c m+(j-c m) r^{-1} r\right] a+\left[c n+(k-c n) s^{-1} s\right] b=j a+k b .
\end{aligned}
$$

But this contradicts Lemma 1.1, hence $N$ is not a $Z S$ near-ring.

\section{Non-Abelian $Z S$ near-rings}

First we consider distributive $Z S$ near-rings. For any distributive nearring $N, A=\{a \in N \mid a x=x a=0$ for all $x \in N\}$ is an ideal containing $N^{\prime}$. Heatherly [3] noted that whenever $N^{2}$ is not contained in $A$, then $N / A$ is a non-trivial zero square ring; hence the limitations of order and nilpotency for zero square rings [8] are inherited by these near-rings. The commutator near-rings constructed by Heatherly on nilpotent-class-two groups and by Feigelstock [1] on generalized nil-2 groups are distributive $Z S$ near-rings with $N^{2} \subseteq A$. The next several results show that such near-rings can be defined on a wide variety of additive groups including all finite nilpotent groups and dihedral groups of order $8 n$ for $n \geq 1$.

Definition 3.1. A finite Abelian group $G$ will be called $k q$-non-cyclic for some prime $q$ and some positive integer $k$ if, when $G$ is written as the direct sum of cyclic groups of prime power order, at least $k$ of the summands are $q$-groups.

THEOREM 3.2. A finite non-Abelian group $N$ is the additive group of a 3nilpotent distributive $Z S$ near-ring if and only if $N$ has a normal subgroup $A$ which contains $N^{\prime}$, there exists a prime $p$ such that $N / A$ is $2 p$-non-cyclic, and $p|| A \mid$.

Proof. (i) Let $N$ be a non-Abelian group with properties described in the theorem, and let $t \in A$ be such that $o(t)=p$. Also let $B$ and $C$ be two summands of $N / A$ which are $p$-groups, $N / A=B \oplus C \oplus G, x \in B$ such that 
$p x \in A$, and $y \in C$ such that $p y \in A$. Then every element of $N$ can be uniquely written as $j x+k y+g+a$ for positive integers $j, k \leq p, g \in G$, and $a \in A$. Define multiplication in $N$ by

$$
\left(j_{1} x+k_{1} y+g_{1}+a_{1}\right)\left(j_{2} x+k_{2} y+g_{2}+a_{2}\right)=\left(j_{1} k_{2}-j_{2} k_{1}\right) t .
$$

It is routine to verify that $(N,+, \cdot)$ is a 3-nilpotent distributive $Z S$ nearring.

(ii) Let $N$ be a finite 3-nilpotent distributive $Z S$ near-ring. Since $N$ is non-trivial, there exists $x, y \in N$ such that $x y \neq 0$; hence there is a prime $p$ such that $p \mid(o(x), o(y))$. It follows that $p|| A \mid, x \notin A$, and $y \notin A$. Suppose $x=j y+a$ for some integer $j$ and some $a \in A$. Then $x y=(j y+a) y=0$. This contradiction implies that $x \notin(j y+A)$ for every integer $j$. Therefore, $x$ and $y$ are in different cyclic summands of $N / A$, and each of these summands has order divisible by $p$. Hence $N / A$ is $2 p$-non-cyclic.

COROLLARY 3.3. Every finite nilpotent group is the additive group of a distributive ZS near-ring.

Proof. Let $N$ be a finite nilpotent group. Then $N=S \oplus G$ where $S$ is a non-Abelian Sylow $p$-subgroup. Let $A$ be the Frattini subgroup of $S$. Then $S^{\prime} \subseteq A, S^{\prime} \neq 0$, and $S / A$ is elementary abelian of order $p^{m}$ with $m>1$ $[2,6]$. It follows that $S / A$ is $2 p$-non-cyclic; so $S$ is the additive group of a distributive $Z S$ near-ring. The direct product of this near-ring and the zero ring on $G$ is a distributive $Z S$ near-ring with additive group $N$.

COROLlaRY 3.4. A dihedral group of order $2 n$ supports a distributive ZS near-ring if and only if $4 \mid n$.

Proof. (i) Let $N$ be a dihedral group of order $2 n$ where $4 \mid n$. Then $N^{\prime}$ has an element of order two and $N / N^{\prime} \cong Z_{2} \oplus Z_{2}$.

(ii) Let $N$ be a dihedral group of order $2 n$ where $4 \nmid n$. If $n$ is even, $N / N^{\prime} \cong Z_{2} \oplus Z_{2}$, but $N^{\prime}$ has no element of order two. IF $n$ is odd, $N / N^{\prime} \cong$ $Z_{2}$. In either case $N$ is not the additive group of a distributive $Z S$ nearring.

The following example shows that there are non-abelian pseudo-distributive $Z S$ near-rings which are not distributive.

EXAmple 3.5. Let $N$ be a dihedral group of order $4 k$ such that $N=$ $\langle a, b\rangle$ where $2 k a=2 b=0$. Every element of $N$ can be uniquely written 
as $j a+m b$ for integers $j, m$ where $0 \leq j<2 k$ and $0 \leq m \leq 1$. So the following multiplication is well-defined on $N$

$$
a(j a+b)=k a \text { and } x y=0 \text { otherwise. }
$$

It is routine to verify that with this multiplication $N$ is a pseudo-distributive $Z S$ near-ring. It is not right distributive since $(a+b) b \neq a b+b^{2}$.

Finally, we consider Feigelstock's question [1]: are there distributively generated $Z S$ near-rings which are not distributive? Although the question remains open, the next theorem places several necessary conditions on such a near-ring.

The following lemmas are stated for reference; the proofs are trivial.

LEMMA 3.6. If $N$ is a near-ring with $N^{+}$generated by a set of right distributive elements whose products commute additively, then $N$ is distributive.

LEMMA 3.7. If $N$ is a near-ring and $a, b \in N$ with $b$ right distributive, then $(-a) b=a(-b)=-(a b)$.

LEMMA 3.8. If $N$ is a ZS near-ring with right distributive elements $a$ and $b$, then $a b=-(b a)$.

THeOREM 3.9. If $N$ is a non-distributive $Z S$ near-ring which is generated additively by a set $D$ of right distributive elements, then

(i) $D$ contains at least three elements;

(ii) if $|N|$ is odd, then $N$ is 3-nilpotent;

(iii) for every $a, b, c \in D, c a+(a b+c b)=(a b+c b)+c a$.

Proof. (i) Suppose $D=\{a, b\}$. Then the only products of elements of $D$ are $0, a b$, and $b a$. Since $a b=-b a$ (Lemma 3.8), all products in $D$ commute additively. Therefore, by Lemma $3.6, N$ is distributive.

(ii) Suppose $N$ is $k$-nilpotent for $k>3$. Then there exist $a, b, c \in D$ such that $a b c \neq 0$. Also

$$
(a+b c)(a+b c)=b c a+a b c=0,
$$

and

$$
(a b+c)(a b+c)=c a b+a b c=0 .
$$

Hence

$$
b c a=c a b=(c a) b=(-b) c a=-(b c a) .
$$

Thus $b c a$ has additive order two, which contradicts the fact that $|N|$ is odd.

(iii) Let $a, b, c \in D$. Then

$$
(a+b+c)(a+b+c)=b a+c a+a b+c b+a c+b c=0 .
$$

Hence $c a+a b+c b=a b+c b+c a$. 


\section{References}

[1] Shalom Feigelstock, 'Generalized nil 2-groups and near-rings', Indian J. Math. 22 (1980), 99-103.

[2] Marshall Hall, Jr., The theory of groups. (The MacMillan Co., New York, 1959).

[3] Henry E. Heatherly, 'Distributive near-rings', Quart. J. Math. Oxford Ser. (2) 24 (1973), 63-70.

[4] Henry E. Heatherly and Steve Ligh, 'Pseudo-distributive near-rings', Bull. Austral. Math. Soc. 12 (1975), 449-456.

[5] Patricia Jones, Distributive near-rings (Thesis, University of Southwestern Louisiana, 1976).

[6] A. G. Kurosh, Theory of groups, Vol. 1 (Chelsea, New York, 1960).

[7] Günter Pilz, Near-rings (North Holland/American Elsevier, Amsterdam, 1977).

[8] Richard P. Stanley, 'Zero Square Rings', Pacific J. Math. 30 (1969), 811-824.

\section{University of Southwestern Louisiana}

Lafayette, Louisiana 70504

USA 